\title{
Study on Knowledge Sharing Behavior of Users in Social Q\&A Community
}

\author{
Shuli Gao, Xinyu Sun, Jianbin Chen, Yanli Guo \\ Business College, Beijing Union University, Beijing, China \\ Email: shuli.gao@buu.edu.cn
}

How to cite this paper: Gao, S. L., Sun, X. Y., Chen, J. B., \& Guo, Y. L. (2020). Study on Knowledge Sharing Behavior of Users in Social Q\&A Community. Journal of Service Science and Management, 13, 688-701. https://doi.org/10.4236/jssm.2020.134043

Received: July 3, 2020

Accepted: August 25, 2020

Published: August 28, 2020

Copyright (อ 2020 by author(s) and Scientific Research Publishing Inc. This work is licensed under the Creative Commons Attribution International License (CC BY 4.0).

http://creativecommons.org/licenses/by/4.0/

\begin{abstract}
Social Q\&A community provides a new way for users to acquire, exchange and share information, so it is of great significance to study user's knowledge sharing behavior in social Q\&A community. Based on the theory of social exchange, this paper takes Zhihu community as the research object, analyzes the factors that affect user's knowledge sharing behavior in the social Q\&A community, and conducts quantitative research through negative binomial regression. The results show that in Zhihu community, self-display, user recognition, social learning and other dimensions have a significant positive impact on user's knowledge sharing behavior, but the degree of impact is different. Finally, according to the empirical results, the paper puts forward relevant suggestions and measures to better promote user's knowledge sharing behavior.
\end{abstract}

\section{Keywords}

Knowledge Sharing Behavior, Q\&A Community, Zhihu Community

\section{Introduction}

With the rapid development of network technology, the Internet application model has undergone major changes, from the Web 1.0 era in which users get access to information of web portals through browsers to the Web 2.0 era in which users are both network visitors and content creators. Knowledge tends to be diversified and fragmented. In many applications of Web 2.0, social Q\&A community has become a way for users to get, communicate and share information, which has a profound impact on people's lifestyle. Social Q\&A refers to an online service where users present their information needs in the form of natural language and respond to others, while a social Q\&A community refers to an online site that provides such services, which has the characteristics of social media, 
social networking sites, and knowledge management system, etc. In the social Q\&A community, users can search for information and knowledge they need, answer questions, express opinions, transform information into knowledge, and thus making users establish social relationships by gathering information.

The social Q\&A community is an important platform for studying the behavior of user information. Both research and practice have shown that the number of users of social Q\&A community has grown rapidly in recent years, such as Quora in the United States and China's Zhihu. Therefore, what factors are driving users to share knowledge in the social Q\&A community? That is, why would community users spend time and energy sharing knowledge with others without any guarantee of immediate financial return? What factors can effectively promote users to actively share knowledge with others in the social Q\&A community? The discussion of this issue will help to reveal the influence mechanism of user behaviors of social Q\&A communities, thus promoting the sustainable development of social Q\&A community.

This paper will take Zhihu, China's largest social Q\&A community, as a research object to conduct an in-depth research on this issue. Founded in 2010, the number of Zhihu users was over 220 million, the number of questions exceeded 30 million, and the number of answers exceeded 130 million as of the end of November 2018 (Chinairn.com, 2018). Zhihu provides a high-quality knowledge sharing platform that allows users to give professional and serious answers when they see problems with the desire to share. In this process, they have gained respect from other users in the community through approval, gratitude, comments, and direct messages, becoming cyber stars or opinion leaders in the professional field and become self-actualized. These factors all increase the willingness of users to share and stimulate their knowledge sharing behavior. This paper will focus on the following two aspects: What factors affect the knowledge sharing behavior of Zhihu users? How do these factors interact with each other? The research will deepen our understanding of knowledge sharing behavior and its causes of the social Q\&A community users, and help the social Q\&A community improve the design and further promote the users' knowledge sharing behavior.

The structure of the paper is as follows: the second part combs the theory used in the research of social Q \& a community users' behavior, and proposes that this study will be based on social exchange theory; the third part puts forward the theoretical framework and research hypothesis of this study; the fourth part will take Zhihu as an example, obtain relevant data through web crawler technology, and use negative binomial regression to test hypothesis; the fifth part is to raise the conclusion and suggestions, and also discuss the innovation, limitations and future prospects of this study.

\section{Literature Review}

Knowledge sharing is one of the important issues in the field of knowledge management research, and has been widely concerned by people. After review- 
ing relevant literatures, it can be found that with the development of economy and society, organizational structure and ways of cooperation among members are changing, influencing factors of knowledge sharing are gradually becoming diversified and complicated, and relevant studies are evolving with the change of times. On the basis of analyzing the characteristics of the social Q\&A community, scholars at home and abroad have applied different theories to discuss user behavior in the social Q\&A community, such as social capital theory, social cognitive theory, perceived value theory, social exchange theory, and theory of motivation, etc.

Based on the theory of social capital, Hsu et al. (2011) studies the knowledge sharing behavior of online community users from the perspectives of structural, relational and cognitive dimensions of social capital. The results show that the interaction of social capital and shared vision can increase the trust among users, and trust can effectively promote the generation of knowledge sharing behavior. Sun et al. (2012) found that the cognitive dimension of social capital had a positive impact on users' knowledge sharing behavior. According to Lin et al. (2017), the dimension of social capital structure is significantly positively correlated with the quality of knowledge sharing, but not significantly correlated with the quantity of knowledge sharing; the cognitive dimension displayed significantly positive correlations with the quality and quantity of knowledge sharing. Some scholars believe that the three dimensions of social capital have a great positive impact on the quality and quantity of knowledge sharing (Cao \& Gu, 2018; Wartburg, Rost, \& Teichert, 2006; Kang et al., 2011; Ye, Xing, \& Kapre, 2017). Most of these studies are based on questionnaires for empirical analysis.

Some scholars also study the influence of cognition on knowledge sharing behavior based on social cognitive theory, mainly from self-efficacy and outcome expectations. Papadopoulos et al. (2013) found that knowledge sharing self-efficacy has a positive impact on the willingness to share knowledge. Jin et al. (2013) explained the impact of user satisfaction and self-efficacy on their willingness to share knowledge from a theoretical and empirical perspective, and pointed out that there is a significant positive relationship between self-efficacy and satisfaction. He and Guo (2016) analyzed the impact of outcome expectations and environmental factors on knowledge sharing in mobile communities, and concluded that outcome expectations are important influencing factors and mediate between environmental factors and behaviors. Luo et al. (2014) and Hua et al. (2019) had published similar studies. These studies also conduct empirical analysis based on questionnaires.

In recent years, scholars have widely used perceived value in the study of knowledge sharing behavior of online community users. Previous studies divide the value perception of user knowledge sharing into practical value, sentimental value, social value and altruistic value. The research of Wolf et al. (2011) shows that perceived benefits and expected return promote users' knowledge sharing to a large extent. Jeong (2012) took social Q\&A community as the research object, and found that compared with other factors, the fun obtained in the process of 
knowledge sharing has a stronger influence on the behavior of knowledge sharing. Lou et al. (2013) argue that the rewards of the reputation system of online Q\&A communities could affect the amount of knowledge sharing, but have no significant impact on quality. Other studies have undertaken similar research (Chiu, Hsu, \& Wang, 2006; Rafaeli, Raban, \& Ravid, 2007; Kim \& Oh, 2009; Alam et al., 2017).

Some scholars believe that the behavior of users' sharing knowledge in online communities is similar to the logic of social exchange in reality. According to Kankanhalli et al. (2005), knowledge contributors can not only derive some new value from the information they provide, but also get some remuneration from others. Based on the social exchange theory, Zhang (2015) confirms that psychological rewards, altruism and other factors have a significant positive impact on the social Q\&A community's willingness to share knowledge. Some scholars believe that users' self-expression degree, peer recognition, and social learning have a significant positive impact on the quantity of knowledge contribution (Choi \& Shah, 2006; Gupta \& Kim, 2007; Jin et al., 2015; Khansa et al., 2015; Xu \& Zhang, 2018).

From the existing literature, based on the social capital theory and social cognitive theory, the social Q\&A community's knowledge sharing behavior is studied mainly through questionnaire survey, and the impact of social Q\&A community on knowledge sharing is analyzed using psychological scale. The practice ignores the effects of the actual behavior characteristics of users in the online community (such as the actual number of questions asked by users, the adoption rate of answers, and comments of netizens). Based on the social exchange theory, the study on the knowledge sharing behavior of social Q\&A communities starts from the user data provided by the platform. Therefore, this paper will study the knowledge sharing behavior of the largest social Q\&A community in China based on social exchange theory, hoping to add necessary supplement to existing research.

\section{Theoretical Model and Research Hypothesis}

In the social Q\&A community, users discover and produce knowledge by focusing on problems, topics, and users, and gradually establish social relationships with other users in the community through knowledge sharing to form a socialized community. Based on the existing literature, this paper will construct the knowledge sharing behavior model of users in the social Q\&A community from the three aspects of self-presentation, user recognition and social learning, and analyze the impact of various factors on the knowledge sharing behavior. The model constructed is shown in Figure 1. Based on the established model, this paper will present the corresponding theoretical assumptions.

\subsection{Users' Self-Presentation and Knowledge Sharing Behavior}

For individuals, "who am I" and "what do I do" are the factors that make up their personal image, which is the key to distinguish them from others. 


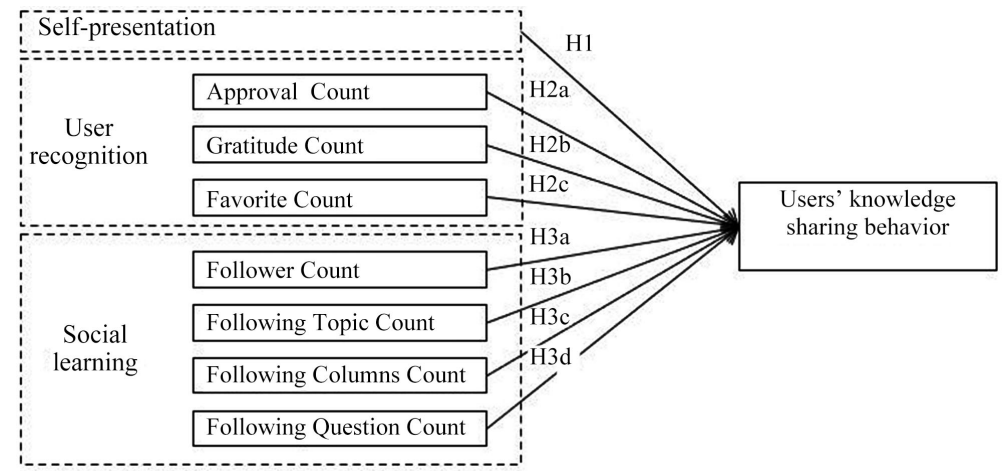

Figure 1. Model of knowledge sharing behavior of users in social Q\&A community.

Self-presentation, also known as self-display, refers to the efforts made by individuals to express and show their own image to others when they start to associate with others, so as to reach mutual understanding with others (Golder \& Donath, 2004). Self-presentation information mainly includes the users' personality, experience, and identity, etc. In social media, users habitually use information about others' self-presentation on the website to infer their status, hobbies and behavior. Kim and Lee (2011) believe that self-presentation could help them obtain more perceived social support and thus finding more happiness. Building a virtual online personal identity not only enhances confidence and reputation, but also amplifies the likelihood of expected returns (Din \& Yang, 2016). These studies show that self-presentation is one of the important factors for users to participate in online community for knowledge sharing. On the Zhihu platform, there are many ways to display oneself by uploading head portraits, editing personal information, and posting live updates, etc. The users' willingness to self-display is likely to affect the willingness to share knowledge. Therefore, this paper proposes the following assumptions:

H1: The willingness to self-display has a significant positive impact on the knowledge sharing behavior of users in the social Q\&A communities.

\subsection{Users' Recognition and Knowledge Sharing Behavior}

Social exchange theory believes that users' behavior is determined by perceived income and perceived cost. Revenue mainly refers to tangible rewards such as respect and reputation. Cost refers to time, money, physical strength and spiritual cost. When the perceived income is greater than the perceived cost, users will participate in knowledge sharing. Therefore, the fundamental driving force for users' knowledge sharing is the expected gains in the process of knowledge sharing. In the community, users' knowledge sharing is recognized by other users as intrinsic rewards, which in turn encourage them to share more knowledge. Such a positive feedback cycle can promote the users' future knowledge sharing behavior (Yao, Tsai, \& Fang, 2015). On the online Q\&A platform such as Zhihu, the users' approval can be seen from gratitude account and favorite count. The more the number of acquisitions, the more users can be recognized, and the stronger willingness of the knowledge sharing behavior. 
Therefore, the following assumptions are made:

H2a: Approval count has a significant positive impact on the knowledge sharing behavior of users in the social Q\&A communities.

H2b: Gratitude count has a significant positive impact on the knowledge sharing behavior of users in the social Q\&A communities.

$\mathrm{H} 2 \mathrm{c}$ : Favorite count has a significant positive impact on the knowledge sharing behavior of users in the social Q\&A communities.

\subsection{Users' Social Learning and Knowledge Sharing Behavior}

According to social cognition theory, the behavior of social members is shaped and constrained by their own cognition and social network. According to social learning theory, many behaviors of social members are learned in the process of observing others' behaviors and determining the external results of behaviors, so the influence of social members on other members' behavior changes can be explained by social learning theory (Anderson, Winett, \& Wojcik, 2007; Porter \& Donthu, 2008). In the social Q\&A community, many behaviors of users are learned in the process of observing the behavior of others and determining the external outcomes of behaviors. Therefore, social learning also reflects the behavioral changes of users to other users (Zhou \& Wang, 2016). In the social Q\&A community, community users can better predict the outcome expectations of their knowledge sharing behavior through social learning, thus affecting their willingness to share knowledge. On the Q\&A platform like Zhihu, social learning can be seen from the number of followers, topics, columns, and questions. The more attention received, the richer the social learning, and more users are willing to share knowledge.

Therefore, the following assumptions are made:

H3a: The number of followers has a significant positive impact on the knowledge sharing behavior of users in the social Q\&A communities.

$\mathrm{H} 3 \mathrm{~b}$ : The number of following topics has a significant positive impact on the knowledge sharing behavior of users in the social Q\&A communities.

$\mathrm{H} 3 \mathrm{c}$ : The number of following Column has a significant positive impact on the knowledge sharing behavior of users in the social Q\&A communities.

H3d: The number of following questions has a significant positive impact on the knowledge sharing behavior of users in the social Q\&A communities.

Based on the user behavior data provided by the platform, the paper obtains the actual behavior characteristics of users in the social Q \& A community through web crawler technology, and quantifies the behavior of self-presentation, user recognition, social learning and user knowledge sharing behavior in the theoretical model, and finally verifies the hypothesis through negative binomial regression.

\section{Empirical Test}

\subsection{Variable Definition}

Zhihu is a well-known social Q\&A community in China. It is a very representative window to understand China's emerging middle class and influential people. 
There are two types of information about user's profile page: personal information and Zhihu community participation information. Personal information includes personal portrait, profile, gender, residence, career experience, education experience, etc. Although users are encouraged to improve their information, this is not necessary. Zhihu community participation information includes all user behavior information, including the number of questions, answers, co-editing, approval count, following topics, following columns and other information.

This paper chooses the platform as a source of users' knowledge-sharing data. The author crawled user data from Zhihu as of October 31, 2018, totaling 100,000 items. The data includes: users' place of residence, users' professional history, education experience, resume, approval account, gratitude account, favorite account, number of co-editing, number of answers, number of questions asked, follower account, number of following topics, number of following columns, and number of following questions.

By sorting out the existing data, it is found that the personal identity attributes of the Zhihu users are: users' place of residence, users' professional history, users' education experience, users' resume, all of which are optional, and the user gets 1 point for each identity attribute. If four identity attributes are filled in, it is 4 points. If no identity attribute is filled in, it is 0 points. In terms of user recognition, it is mainly measured from approval count, gratitude count, and favorite count. In terms of social learning, it is mainly measured from the number of followers, topics, columns, and questions. For the knowledge sharing behavior, the number of co-edits, answers and questions are added as the data to measure the knowledge sharing behavior.

Table 1 is a list of variables that reflect the measured items, measured variables, and interpretations in this study.

\subsection{Data Analysis}

Table 2 is a descriptive statistics list of variables including variable name, sample size, mean value, standard deviation, minimum value, and maximum value. As can be seen from Table 2, among the eight independent variables, the mean of approval count is the biggest, the mean of following Column count is the smallest, the standard deviation of favorited count and follower count are relatively high. For dependent variable of knowledge sharing behavior, the mean is 93.12, the $\min$ is 0 and the $\max$ is 152,133 .

The distribution of the knowledge sharing behavior is listed in Table 3. The value of knowledge sharing behavior is mainly concentrated in $0-10$, with a total of $45.6 \%$, and $45.4 \%$ concentrated in $11-150$. There are few users whose knowledge sharing behavior exceeds 150. According to the distribution of knowledge sharing behavior, it can be seen that the distribution of knowledge sharing behavior does not conform to the normal distribution, thus the linear regression cannot be used. Since the dependent variable knowledge sharing behavior is a counting variable, the variable of non-negative integer value. At least a part of the values is 0 , therefore, a count-based model is considered. 
Table 1. List of variables.

\begin{tabular}{|c|c|c|}
\hline Measured items & Measured variables & Interpretations \\
\hline Self-presentation & Identity & Score of self-presentation \\
\hline \multirow{3}{*}{ User recognition } & Approval Count & Approval number \\
\hline & Gratitude Count & Gratitude number \\
\hline & Favorite Count & Favorite number \\
\hline \multirow{4}{*}{ Social learning } & Follower Count & Number of followers \\
\hline & Following Topic Count & Number of Following topics \\
\hline & Following Column Count & Number of Following columns \\
\hline & Following Question Count & Number of Following questions \\
\hline $\begin{array}{c}\text { Knowledge sharing } \\
\text { behavior }\end{array}$ & Knowledge Sharing Behavior & $\begin{array}{c}\text { Number of co-editing }+ \text { Number of answers }+ \\
\text { Number of questions asked }\end{array}$ \\
\hline
\end{tabular}

Table 2. Descriptive statistics of variables.

\begin{tabular}{cccccc}
\hline Variable name & Sample size & Mean value & SD & Min. & Max. \\
\hline Identity & 100,000 & 1.28 & 1.26 & 0 & 4 \\
Approval Count & 100,000 & 9877.05 & 9999.74 & 0 & 29,999 \\
Gratitude Count & 100,000 & 706.24 & 5004.94 & 0 & 362,156 \\
Favorited Count & 100,000 & 2266.55 & $18,869.23$ & 0 & $1,484,682$ \\
Follower Count & 100,000 & 1577.72 & $14,458.44$ & 0 & $1,053,750$ \\
Following Topic Count & 100,000 & 42.03 & 117.26 & 0 & 7977 \\
Following Column Count & 100,000 & 10.98 & 39.02 & 0 & 2474 \\
Following Question Count & 100,000 & 239.91 & 1791.06 & 0 & 471,470 \\
knowledge sharing behavior & 100,000 & 93.12 & 955.56 & 0 & 152,133 \\
\hline
\end{tabular}

Table 3. Frequency of variables of knowledge sharing behavior.

\begin{tabular}{cccc}
\hline No. & Frequence & Percentage & Cumulative percentage\% \\
\hline 0 & 12,914 & 12.9 & 12.9 \\
1 & 6667 & 6.7 & 19.6 \\
2 & 4887 & 4.9 & 24.5 \\
3 & 3862 & 3.9 & 28.3 \\
4 & 3268 & 3.3 & 31.6 \\
5 & 2946 & 2.9 & 34.5 \\
6 & 2629 & 2.6 & 37.2 \\
7 & 2445 & 2.4 & 39.6 \\
8 & 2168 & 2.2 & 41.8 \\
9 & 1961 & 2.0 & 43.7 \\
10 & 1822 & 1.8 & 45.6 \\
$11-150$ & 9014 & 45.4 & 91.0 \\
More than 150 & & 9 & 100.0 \\
\hline
\end{tabular}


The basic count-based model is the Poisson regression model, which assumes that the distribution of the dependent variable is the Poisson distribution, that is, the mean is equal to the standard deviation. As seen in Table 2 , the mean value of the knowledge sharing behavior is 93.12, and the standard deviation is 955.56. The mean value is not equal to the standard deviation. Obviously, the Poisson model is not suitable for this over-discrete problem. Therefore, negative binomial regression model will be used in data analysis.

It can be seen from the above statistical analysis that the distribution of the original data is highly skewed, so all variables will be logarithmic in the process of data analysis. Because some original data is zero, we will add 1 to the data and do logarithmic processing according to the relevant literature. The results are shown in Table 4.

It can be seen from Table 4 that the identity variable has a significant positive influence on the knowledge sharing behavior (coef. $=0.2591, p$-value $<0.01$ ); approval count has a significant positive influence on the knowledge sharing behavior (coef. $=0.1205, p$-value $<0.01$ ); gratitude count, favorite count, follower count, following topic count, following column count, following question are also have the significant positive effect on the knowledge sharing behavior. Among them, the number of concerns has the greatest impact on knowledge sharing behavior, followed by identity, while favorite count has the least impact on knowledge sharing behavior.

In conclusion, hypothesis $\mathrm{H} 1$ explores whether the willingness of self-presentation has an impact on the knowledge sharing behavior of users on the social Q\&A platform. Analysis results show that the identity variable has a significant positive impact on the knowledge sharing behavior, so this hypothesis is supported. The significant positive impact of the identity variable indicates that when users show more personal information in the social Q\&A community, they are more willing to share their knowledge in the community. Therefore, hypothesis $\mathrm{H} 1$ is supported.

Table 4. Analysis result of negative binomial regression.

\begin{tabular}{|c|c|c|}
\hline Ln_Knowledge Sharing & Coef. & Std. Err \\
\hline ln_Indentity & $0.2591^{* *}$ & 0.0062 \\
\hline ln_Approval Count & $0.1205^{\star *}$ & 0.0022 \\
\hline Ln_GratitudeCount & $0.2929^{* *}$ & 0.0039 \\
\hline ln_FavoriteCount & $0.0166^{* *}$ & 0.0036 \\
\hline ln_Follower Count & $0.0953^{* *}$ & 0.0032 \\
\hline ln_FollowingTopic Count & $0.0349^{* *}$ & 0.0031 \\
\hline ln_FollowingColumn Count & $0.1424^{* *}$ & 0.0031 \\
\hline ln_FollowingQuestion Count & $0.3566^{* *}$ & 0.0034 \\
\hline _cons & $-0.7889^{* *}$ & 0.0055 \\
\hline Wald $\mathrm{Chi}^{2}$ & \multicolumn{2}{|c|}{7848.27} \\
\hline Pesudo $\mathrm{R}^{2}$ & \multicolumn{2}{|c|}{0.1260} \\
\hline Number of Obs & \multicolumn{2}{|c|}{100,000} \\
\hline
\end{tabular}

a. ${ }^{*} p<0.05,{ }^{* *} p<0.01$. 
Hypotheses $\mathrm{H} 2 \mathrm{a}$ and $\mathrm{H} 2 \mathrm{~b}$ explore whether users' approval has an impact on the knowledge sharing behavior of users on the social Q\&A platform. The analysis results show that the approval count, gratitude count and favorite count all have a significant positive impact on the knowledge sharing behavior, indicating that the more approval, gratitude and collection obtained in the social Q\&A community, the more they are willing to share their knowledge with other users. Therefore, $\mathrm{H} 2 \mathrm{a}, \mathrm{H} 2 \mathrm{~b}$, and $\mathrm{H} 2 \mathrm{c}$ are supported.

Hypotheses H3a, H3b, H3c, and H3d explore whether social learning has an impact on the knowledge sharing behavior of users on the social Q\&A platform. The analysis results show that the follower count, following topic count, following column count and following question count of the social learning have a significant positive impact on the knowledge sharing behavior, indicating that when the users are willing to conduct more social learning in different ways in the social Q\&A community, it is more likely to stimulate their thoughts and share knowledge with other users in the community. Therefore, the assumptions H3a, $\mathrm{H} 3 \mathrm{~b}, \mathrm{H} 3 \mathrm{c}$ and $\mathrm{H} 3 \mathrm{~d}$ are supported.

\section{Conclusion and Suggestions}

\subsection{Conclusion}

This paper explores the influence of social Q\&A community users' self-presentation, user recognition, and social learning on users' knowledge sharing behavior. The following conclusions are drawn from this study.

The willingness of self-presentation has a significant positive impact on the knowledge sharing behavior of users in the social Q\&A community. This study measures the users' self-presentation willingness according to the degree of personal information displayed. It demonstrates that the richer the personal information, the stronger the self-presentation willingness, and thus promoting the users' knowledge sharing behavior on the Zhihu platform. As far as user recognition is concerned, the number of gratitude and collection won by the users can promote the users' knowledge sharing in the social Q\&A community. When a user receives gratitude and collection, it means that other users have affirmed him, this move has greatly stimulated the user to continue to shares his knowledge on Zhihu. Social learning has a significant positive impact on the knowledge sharing behavior of users in the social Q\&A community. In the community, social learning can be carried out in daily communication. Through learning, users can be more willing to share personal opinions. While sharing, other users can also implement social learning, which is a virtuous circle. It can be seen that the users' social learning greatly promotes the users' knowledge sharing behavior on the Zhihu platform.

\subsection{Suggestions}

Zhihu users accumulate social recognition mainly through Q \& A. even the ordinary people can obtain good social recognition as long as they continuously 
share high-quality knowledge content. But in the survey, we found that a small number of users communicate in the social Q\&A community with the attitude of answering doubts and helping each other, and a large part of users are for the purpose of leisure time and browsing aimlessly, and they are more inclined to browse webpage rather than answer questions in the community. Combined with the conclusions of the study, in order to promote users in social Q\&A community, this paper puts forward following suggestions:

First of all, Zhihu should build a high-quality Q\&A community to build trust relationship among users and help users share knowledge more fully. Knowledge sharing behavior is based on mutual communication. Social Q\&A community operators should not only consider the realization of users' self-interest, but also consider how to help users realize their altruistic interests. By setting up the corresponding incentive mechanism, users are encouraged to create activities and participate in activities, so as to fully realize the altruistic interests of users. In this way, community can build a strong relationship network and reduce the resistance of knowledge sharing.

Second, the social Q\&A community can encourage users to register by real name through mobile phone number, ID card, education background, etc., so as to make the community environment more authentic and credible, and make users feel that they can trust each other. Once users perceive information security, more users will be willing to share with each other and enhance their desire for knowledge sharing.

Finally, Q\&A community can regularly invite experts and professors in various fields to conduct online lectures, knowledge lectures or award-winning answering activities in the community. Community can also invite the event parties, specific companies, products, etc. to take part in Q\&A activities, so as to provide more objective and real answers from the first perspective. In addition, for those who have worked in a certain field for many years, but have not yet reached the level of experts, they should be also actively encouraged to take part in community Q\&A, which is a key link to improve their knowledge and activity. Such groups will strive to participate in knowledge sharing activities in order to gain more social recognition and promote to a higher level.

\subsection{Research Contributions}

Based on the theory of social exchange, the paper studies the knowledge sharing behavior of the largest social Q\&A community in China, and constructs a knowledge sharing behavior model of users from three aspects. The paper reveals the influence and interaction of various factors on knowledge sharing behavior, enriches the existing literatures of knowledge sharing behavior of users in social Q\&A community.

Different from the way of obtaining data from the questionnaire, the paper uses web crawler technology to obtain the actual behavior characteristics of users in the social Q\&A community, and combines the theoretical model with nega- 
tive binomial regression to conduct empirical analysis, which provides a new idea for quantifying user knowledge sharing behavior.

\subsection{Research Limitations and Future Prospects}

This paper is to analyze the influence mechanism of knowledge sharing behavior of users in social Q\&A community, but there are still some shortcomings. Firstly, in the study of knowledge sharing behavior of users in social Q\&A community, Zhihu is chosen as the research object, and the data source is relatively single. Secondly, the paper mainly uses the platform user behavior data for research, and the quantification of each dimension in the model can also be measured and explained by more abundant indicators, such as knowledge sharing behavior, in addition to considering the amount of knowledge sharing, the quality of knowledge sharing can also be considered.

In the follow-up research, we need to combine semantic web, machine learning and other technologies to further strengthen the evaluation of the quality of user generated answers, and further explore the impact of different dimensions on the quality of answers. At the same time, the knowledge sharing behavior of users in different social Q\&A communities can be compared and analyzed to study the similarities and differences of knowledge sharing behaviors in different communities.

\section{Acknowledgements}

This paper was supported by Beijing Philosophy and Social Science Research Program (17BGL033) (15JGC163), and Premium Funding Project for Academic Human Resources Development in Beijing Union University (BPHR2017CS06), (BPHR2018AS01).

\section{Conflicts of Interest}

The authors declare no conflicts of interest regarding the publication of this paper.

\section{References}

Alam, A., Khusro, S., Ullah, I., \& Karim, M. S. (2017). Confluence of Social Network, Social Question and Answering Community, and User Reputation Model for Information Seeking and Expert's Generation. Journal of Information Science, 43, 260-274. https://doi.org/10.1177/0165551516637322

Anderson, E. S., Winett, R. A., \& Wojcik, J. R. (2007). Self-Regulation, Self-Efficacy, Outcome Expectations, and Social Support: Social Cognitive Theory and Nutrition Behavior. Annals of Behavioral Medicine, 34, 304-312. https://doi.org/10.1007/BF02874555

Cao, L., \& Gu, B. G. (2018). Analysis of the Effect of Social Capital on Knowledge Sharing in the Context of Social Networking. Journal of Modern Information, 38, 41-47.

Chinairn.com (2018). http://www.chinairn.com/hyzx/20181213/13481222.shtml

Chiu, C., Hsu, M., \& Wang, E. (2006). Understanding Knowledge Sharing in Virtual Communities: An Integration of Social Capital and Social Cognitive Theories. Decision Support Systems, 42, 1872-1888. https://doi.org/10.1016/j.dss.2006.04.001 
Choi, E., \& Shah, C. (2006). User Motivations for Asking Questions in Online Q\&A Services. Journal of the Association for Information Science and Technology, 67, 1182-1197. https://doi.org/10.1002/asi.23490

Din, D. H., \& Yang, Z. B. (2016). The Influencing Factors in Knowledge Sharing of Social Question-and-Answer Community: A Case Study of Zhihu. Journal of Management Case Studies, 9, 212-223.

Golder, S. A., \& Donath, J. (2004). Social Roles in Electronic Communities. In Proceedings of the International Conference of Internet Research (pp. 13-22). Brighton, England.

Gupta, S., \& Kim, H. W. (2007). Developing the Commitment to Virtual Community: The Balanced Effects of Cognition and Affect. Information Resources Management Journal, 20, 28-45. https://doi.org/10.4018/irmj.2007010103

He, D. D., \& Guo, D. Q. (2016). Research on the Influencing Factors of Individual Knowledge Contribution in Mobile Community Based on Social Cognitive Theory. Theory \& Application, 39, 82-89.

Hsu, M., Chang, C. M., \& Yen, C. H. (2011). Exploring the Antecedents of Trust in Virtual Communities. Behavior \& Information Technology, 30, 587-601. https://doi.org/10.1080/0144929X.2010.549513

Hua, H. S., Zhang, F., Zhang, J. L., Li, M. L., \& Han, R. Z. (2019). Social Cognition Theory-Based Factors Influencing Continuing Use Behaviors of Online Health Community Users. Chinese Journal of Medical Library and Information, 28, 56-62.

Jeong, O. R., \& Oh, J. (2012). Social Community-Based Blog Search Framework. In International Conference on Database Systems for Advanced Applications (pp. 130-141). Berlin: Springer. https://doi.org/10.1007/978-3-642-29023-7 15

Jin, J. H., Li, Y. J., Zhong, X. J., \& Li, Z. (2015). Why Users Contribute Knowledge to Online Communities: An Empirical Study of an Online Social Q\&A Community. Information \& Management, 52, 840-849. https://doi.org/10.1016/j.im.2015.07.005

Jin, X. L., Zhou, Z., Lee, M. K. O., \& Cheung, C. M. K. (2013). Why Users Keep Answering Questions in Online Question Answering Communities: A Theoretical and Empirical Investigation. International Journal of Information Management, 33, 93-104. https://doi.org/10.1016/j.ijinfomgt.2012.07.007

Kang, M., Kim, B., Gloor, P., \& Bock, G. W. (2011). Understanding the Effect of Social Networks on User Behaviors in Community-Driven Knowledge Services. Journal of the Association for Information Science and Technology, 62, 1066-1074. https://doi.org/10.1002/asi.21533

Kankanhalli, A., Tan, B. C. Y., \& Wei, K. K. (2005). Contributing Knowledge to Electronic Knowledge Repositories: An Empirical Investigation. MIS Quarterly, 29, 113-143. https://doi.org/10.2307/25148670

Khansa, L., Ma, X., Liginial, D., \& Kim, S. S. (2015). Understanding Members' Active Participation in Online Question-and-Answer Communities: A Theory and Empirical Analysis. Journal of Management Information Systems, 32, 162-203. https://doi.org/10.1080/07421222.2015.1063293

Kim, J., \& Lee, J. R. (2011). The Facebook Paths to Happiness: Effects of the Number of Facebook Friends and Self-Presentation on Subjective Well-Being. Cyberpsychology Behavior and Social Networking, 14, 359-364. https://doi.org/10.1089/cyber.2010.0374

Kim, S., \& Oh, S. (2009). Users' Relevance Criteria for Evaluating Answers in a Social Q\&A Site. Journal of the Association for Information Science and Technology, 60, 716-727. https://doi.org/10.1002/asi.21026 
Lin, L., Xie, L. L., \& He, J. H. (2017). The Empirical Research on the Effect of Social Capital on the Knowledge Sharing Result within the Social Networks-A Research Based on Zhihu. Science \& Technology Progress and Policy, 34, 127-135.

Lou, J., Fang, Y., Lim, K. H., \& Peng, J. Z. (2013). Contributing High Quantity and Quality Knowledge to Online Q\&A Communities. Journal of the Association for Information Science and Technology, 64, 356-371. https://doi.org/10.1002/asi.22750

Luo, H. Y., Lin, X. Y., Gao, C. J., \& Zhu, Z. H. (2014). Empirical Research on Influencing Factors of Knowledge Sharing in Virtual Scientific Research Team. Journal of Modern Information, 34, 20-24.

Papadopoulos, T., Stamati, T., \& Nopparuch, P. (2013). Exploring the Determinants of Knowledge Sharing via Employee Weblogs. International Journal of Information Management, 33, 133-146. https://doi.org/10.1016/j.ijinfomgt.2012.08.002

Porter, C. E., \& Donthu, N. (2008). Cultivating Trust and Harvesting Value in Virtual Communities. Management Science, 54, 113-128.

https://doi.org/10.1287/mnsc.1070.0765

Rafaeli, S., Raban, D. R., \& Ravid, G. (2007). How Social Motivation Enhance Economic Activity and Incentives in the Google Answers Knowledge Sharing Market. International Journal of Knowledge and Learning, 3, 1-11. https://doi.org/10.1504/IJKL.2007.012598

Sun, Y. Q., Fang, Y. L., Lim, K., \& Straub, D. (2012). User Satisfaction with Information Technology Service Delivery: A Social Capital Perspective. Information Systems Research, 23, 1195-1211. https://doi.org/10.1287/isre.1120.0421

Wartburg, I. V., Rost, K., \& Teichert, T. (2006). The Creation of Social and Intellectual Capital in Virtual Communities of Practices: Shaping Social Structure in Virtual Communities of Practice. International Journal of Learning and Change, 1, 299-316. https://doi.org/10.1504/IJLC.2006.010972

Wolf, S., Haefliger, S., \& Spaeth, S. (2011). Participation in Intra-Firm Communities of Practice: A Case Study from the Automotive Industry. Journal of Knowledge Management, 15, 22-39. https://doi.org/10.1108/13673271111108675

$\mathrm{Xu}$, P., \& Zhang, D. (2018). A Research on the Motivation of Knowledge Sharing in Online Q\&A Community: From the Perspective of Social Exchange Theory. Documentation, Information \& Knowledge, 2, 105-112.

Yao, C. Y., Tsai, C. C., \& Fang, Y. C. (2015). Understanding Social Capital, Team Learning, Members' e-Loyalty and Knowledge Sharing in Virtual Communities. Total Quality Management \& Business Excellence, 26, 619-631.

https://doi.org/10.1080/14783363.2013.865918

Ye, D., Xing, Z., \& Kapre, N. (2017). The Structure and Dynamics of Knowledge Network in Domain-Specific Q\&A Sites: A Case Study of Stack Overflow. Empirical Software Engineering, 22, 375-406. https://doi.org/10.1007/s10664-016-9430-Z

Zhang, Y. J. (2015). Research on Knowledge Sharing Behavior in Virtual Community Based on the Social Exchange Theory. Taiyuan: North Central University.

Zhou, T., \& Wang, C. (2016). Research on the Continuous Usage of Knowledge Community Users Based on Social Cognitive Theory. Journal of Modern Information, 9, 82-87. 